\title{
Evolution de l'ostéonécrose des maxillaires chez les patients sous bisphosphonates : à propos d'un cas
}

\author{
Evolution of jaw osteonecrosis in patients treated by bisphosphonates: \\ a case report
}

GUILLAUME RONDOT, ANNE-LAURE EJEIL

\begin{abstract}
RÉSUMÉ
Les indications médicales d'un traitement par bisphosphonates sont nombreuses, en particulier pour le traitement des lésions osseuses de la maladie de Kahler. Cependant il existe des effets secondaires bucco-faciaux de ces médicaments. Le plus largement décrit est l'ostéonécrose des maxillaires.

Le cas présenté est celui d'un patient âgé de 92 ans, atteint d'un myélome multiple de stade III. II consulte initialement pour une gêne causée par le port de son stellite mandibulaire. L'examen endo-buccal révèle la présence d'une érosion muqueuse en « $V$ » sur le versant vestibulaire de la crête alvéolaire mandibulaire antérieure édentée ainsi qu'une ulcération avec exposition osseuse maxillaire postérieure droite. Le patient est traité depuis plus de un an par des cures mensuelles de zolédronate. Ce tableau clinique fait évoquer une ostéonécrose liée à l'administration de bisphosphonates.

Compte-tenu de l'âge du patient, du contexte médical, et de l'absence de gêne fonctionnelle, une intervention chirurgicale n'est pas proposée. Une antibiothérapie est mise en place, associée à une antisepsie locale. La prothèse mandibulaire est ajustée. L'ostéonécrose progresse néanmoins rapidement, aussi bien au maxillaire qu'à la mandibule.

La prise en charge de ces complications demeure problématique et le traitement chirurgical difficile tant le résultat est incertain. L'accent doit donc être mis sur les mesures préventives et conservatrices pour l'ensemble des patients traités par bisphosphonates, car leur efficacité thérapeutique semble incontestable. Med Buccale Chir Buccale 2007; 13 : 153-159.
\end{abstract}

médecine buccale chirurgie buccale

VOL. $13, \mathrm{~N}^{\circ} 3$ 2007

page 153

mots clés: ostéonécrose, bisphosphonates, myélome multiple

\section{SUMMARY}

Medical indications for bisphosphonates treatment are numerous, specifically the management of bone complications in Kahler disease. However, oral complications can be induced by these drugs. The most described complications is the risk of osteonecrosis of the jaw.

This article is about a 92 years old man, affected by a stage 3 of multiple myeloma. The purpose of his first visit is a pain induced by his mandibullary prosthesis. The intra-oral examination shows an erosion on the anterior exside mandibullary crest and an ulceration with necrotic bone exposure on the right posterior maxillary site. The patient is treated for more than one year with alendronate. An osteonecrosis induced by bisphosphonate treatment is diagnosed. Considering the patient's age, the medical context and no functional disturbing, a surgical therapy is contraindicated. An antibiotherapy is prescribed followed by irrigation with chlorhexidine. The mandibullary prosthesis is adjusted. Nevertheless, the bone exposure extension is fast on the maxillary bone as much as on the mandibullary bone. The treatment of osteonecrosis is difficult and surgical therapy is complex with an unprobably healing. As the efficacity of bisphosphonate treatment has been proved, it is fundamental to maintain preventive and preservative therapies. Med Buccale Chir Buccale 2007; 13: 153-159.

key words: osteonecrosis, bisphosphonates, multiple myeloma

Département de Chirurgie Buccale Service d'Odontologie de l'Hôpital Bretonneau 2 rue Carpeaux 75018 Paris

Demande de tirés à part:

Guillaume Rondot fgrondot@neuf.fr 
médecine buccale chirurgie buccale

VOL. $13, \mathrm{~N}^{\circ} 3$ 2007

page 154
En 2005, l'AFSSAPS éditait une lettre d'information destinée aux praticiens susceptibles de prescrire des bisphosphonates (BP) ou de devoir intervenir chirurgicalement sur des patients traités ${ }^{[1]}$. Les premières publications traitant de complications maxillaires imputées à la prise de BP datent de $2003^{[2,3]}$ et, depuis cette date, la littérature foisonne d'articles centrés sur les ostéochimionécroses maxillaires d'origine médicamenteuse ${ }^{[4,5]}$. II s'agit le plus souvent de la description de cas cliniques, le mécanisme physiologique de ces phénomènes demeurant encore méconnu. Malheureusement, il n'existe toujours pas à ce jour de consensus quant à la bonne prise en charge de ces patients. Cependant certains auteurs suggèrent une conduite à tenir sur la base de leurs expériences cliniques.

II ressort de la littérature que tous les patients traités par BP doivent, sans exception, être considérés comme des patients à risque. La prise en charge d'une ostéonécrose déclarée s'avère tellement compliquée que tout doit être mis en œuvre pour l'éviter.

Le but de cet article est donc d'insister sur la nécessité de la prévention et de la connaissance du contexte médical et médicamenteux des patients. Nous le ferons en rapportant un cas clinique où le contexte médical, la rapidité et la diversité d'apparition des lésions nous laissent impuissants face à l'évolution du phénomène de nécrose osseuse.

\section{CAS CLINIQUE}

MrX., 92 ans, se présente pour la $1^{\text {re }}$ fois dans le Service d'Odontologie de l'hôpital Bretonneau en février 2006. II consulte en urgence pour une douleur et une instabilité de sa prothèse mandibulaire sur châssis. L'entretien médical étant délicat et imprécis, la prise de contact avec les médecins traitants est nécessaire afin de mieux évaluer son état de santé. Le patient est suivi pour un myélome multiple à Ig G Kappa de stade III depuis 2003, découvert à la suite d'une fracture vertébrale spontanée.

Son traitement curatif a associé une chimiothérapie selon le schéma Alexanian : Melphalan ${ }^{\circledR}+$ Cortancy ${ }^{\circledR}$ et une radiothérapie des foyers dorso-lombaires [6].
Lors de la première consultation odontologique, le traitement médicamenteux suivi par le patient est le suivant :

- Néorécormion ${ }^{\circledR}$, Tardyféron ${ }^{\circledR}$ et Spéciafoldine ${ }^{\circledR}$ pour traiter l'anémie,

- Forlax ${ }^{\circledR}$, Efferalgan ${ }^{\circledR}$ et Renutery ${ }^{\circledR}$ comme traitement adjuvant,

- Cortancyl ${ }^{\circledR}(5 \mathrm{mg} / \mathrm{j})$ associé à Inipomp ${ }^{\circledR}$,

- Zométa ${ }^{\circledR}$ par cure mensuelle de 4 mg en IV depuis

1 an ${ }^{1 / 2}$ pour le traitement des complications osseuses du myélome.

Le jour de la consultation, le patient présente une asthénie marquée et une dénutrition responsable d'une perte de poids progressive mais il n'existe pas de troubles moteurs. Le bilan hépatique est normal. Une insuffisance rénale, fréquente dans le myélome multiple, complique la situation. Le bilan biologique fait état :

- d'une anémie avec un taux d'hémoglobine à $10,4 \mathrm{~g} \cdot \mathrm{dl}^{-1}$

- d'une inflammation avec une VS à $100 \mathrm{~mm}$ à la première heure et CRP à $61 \mathrm{mg} . \mathrm{I}^{-1}$,

- d'une hémostase perturbée avec un TCA ratio à 0,81 et un TP de $61 \%$,

- d'une numération plaquettaire et leucocytaire normales,

- d'un bilan hépatique normal,

- d'une insuffisance rénale avec des protides totaux à $131 \mathrm{~g} \cdot \mathrm{L}^{-1}$, un taux d'urée à $18,4 \mathrm{mmol} . \mathrm{I}^{-1}$ et de créatinine à $187 \mu \mathrm{mol} . .^{-1}$

Dans les antécédents bucco-dentaires, il est important de noter que les extractions de 31 et 41 ont été réalisées en mai 2005 et celle de 17 en juillet 2005. Un projet de réhabilitation par prothèse implanto-portée avait été proposé à la mandibule, mais le patient l'a refusé. L'examen clinique exobuccal pratiqué initialement ne révèle ni la présence d'adénopathies cervico-faciales ni l'existence de troubles sensitifs. L'examen endobuccal permet de constater à la mandibule l'existence d'une érosion muqueuse sur le versant vestibulaire de la crête mandibulaire édentée dans la région de 32, responsable de douleurs (Fig. 1). Au maxillaire on note une ulcération avec exposition d'os nécrosé (de consistance "sucre mouillé ") dans la région de l'alvéole de 17 non cicatrisée et sur le versant vestibulaire de cette zone. II existe une suppuration, mais le patient ne ressent aucune douleur maxillaire 


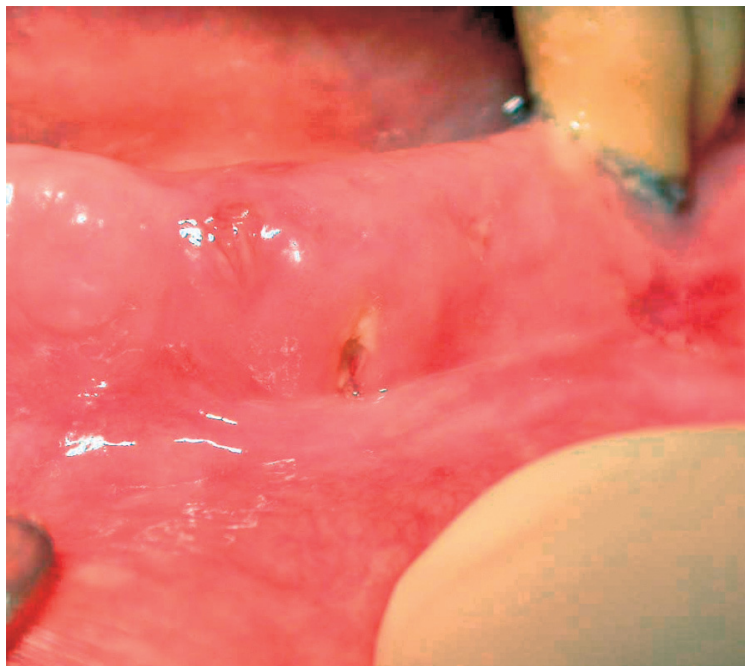

Figure 1: $1^{\text {re }}$ consultation (février 2006), érosion muqueuse en $\mathrm{V}$ sur la crête alvéolaire mandibulaire antérieure

First visit (2006, febrary), mucosa erosion on the mandibular exside anterior crest.

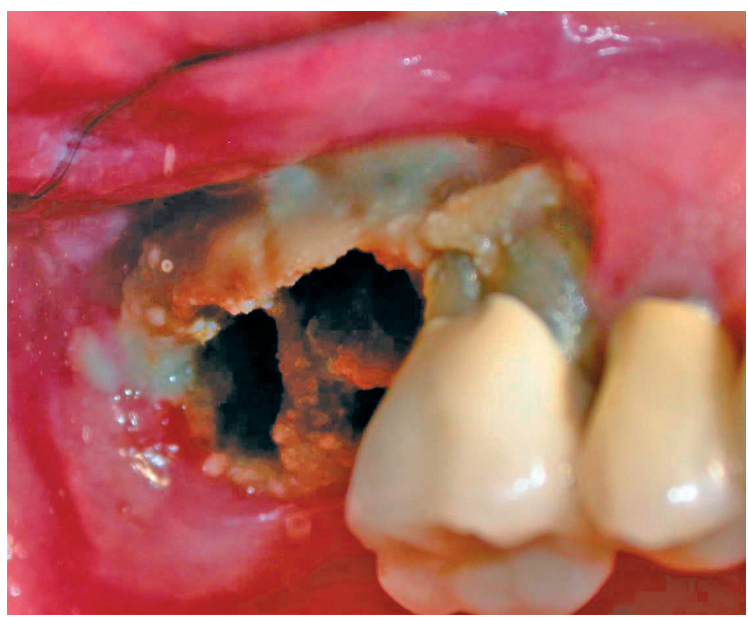

Figure $2: 1^{\text {re }}$ consultation (février 2006), ulcération avec exposition d'os nécrosé dans la région de 16 et 17 absentes.

First visit (2006, febrary), ulceration with necrotic bone exposure on the maxillary first molar and the extracted second molar.

(Fig. 2). Les dents 15 et 16 présentent une mobilité de stade III. La radiographie panoramique permet d'objectiver des zones de raréfaction osseuse dans la région de 16 et 17 . L'hypothèse diagnostique formulée est celle d'une ostéonécrose d'origine médicamenteuse liée au traitement par BP (Zométa $\left.{ }^{\circledR}\right)$. Pour écarter le diagnostic d'une éventuelle localisation du myélome, une biopsie est réalisée. Elle permet de confirmer la nature nécrotique de la pièce opératoire.

Le bilan d'extension est fait après réalisation d'un examen tomodensitométrique maxillo-mandibulaire. Le sinus maxillaire droit apparaît comblé et il existe une hypodensité osseuse autour des racines de 15 et 16 pouvant expliquer leur mobilité (Fig. 3). A la mandibule, il révèle la perte de la corticale externe en regard des sites de 31 et 43. L'examen radiographique des premières vertèbres cervicales montre la présence de lacunes à l'emporte-pièce ayant l'aspect spécifique des lésions du myélome multiple. La scintigraphie osseuse, réalisée par le rhumatologue, permet d'objectiver des zones de remaniement important maxillaire et mandibulaire. Dans un premier temps, une déclaration est faite à un centre de pharmaco-vigilance. Le rhumatologue du patient est contacté, il décide de remplacer le Zométa ${ }^{\circledR}$ par un traitement per os (Clastoban ${ }^{\circledR}$ ). Compte tenu de la suppuration maxillaire, une anitbiothérapie associant pristinamycine et métronidazole est prescrite pendant 15 jours en plus des bains de bouche à la chorhexidine. La prothèse mandibulaire est équilibrée, évidée et rebasée à l'aide d'une résine souple (Viscoge $\left.{ }^{\circledR}\right)$. II est demandé au patient de limiter au maximum le port de son stel-

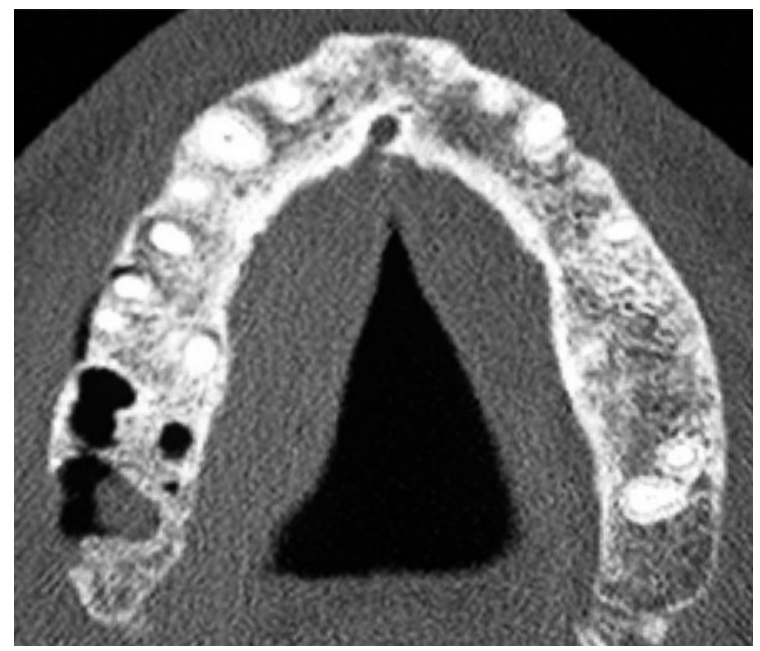

Figure 3 : Examen tomodensitométrique du maxillaire, (coupe axiale). Ostéonécrose étendue au maxillaire postérieur droit avec élargissement desmodontal sur 16 et 15.

Maxillary CT scan, axial view osteonecrosis extented to the right posterior maxillary side. Increased dentoalveolar ligaments on the maxillary first molar and second premolar sites. médecine

buccale

chirurgie

buccale

VOL. $13, \mathrm{~N}^{\circ} 3$

2007

page 155 


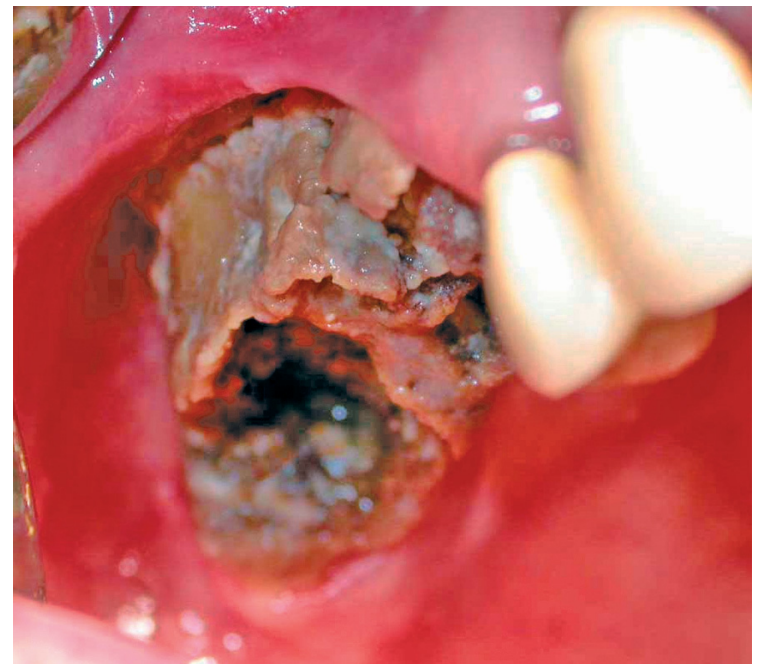

Figure 4 : Aspect clinique (janvier 2007). Perte spontanée de 16 et 15 avec extension de l'exposition osseuse.

Clinical view (2007, january). Spontaneous loss of the first molar and the second premolar with bone expo-

médecine

buccale

chirurgie

buccale

VOL. $13, \mathrm{~N}^{\circ} 3$ 2007

page 156 sure extension.

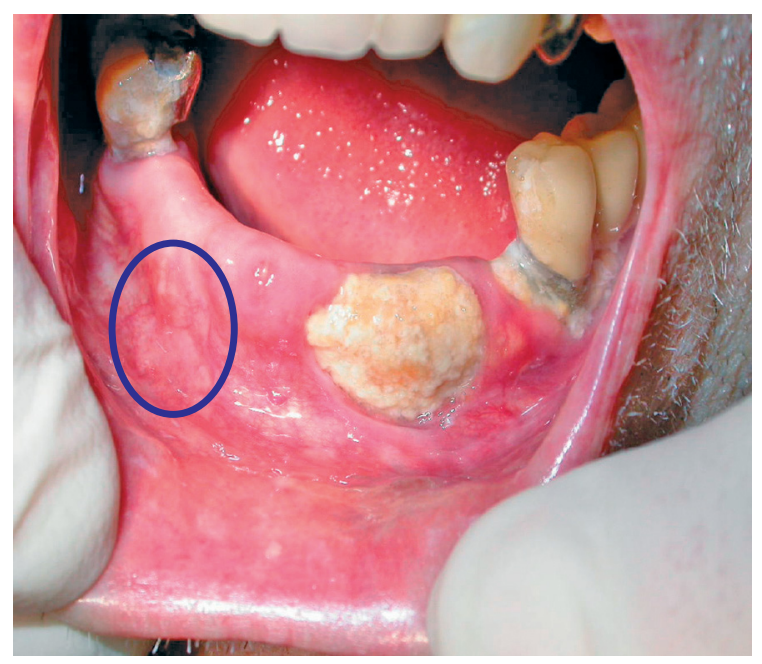

Figure 5 : Aspect clinique de la région mandibulaire (janvier 2007). Extension de l'ostéonécrose mandibulaire avec atteinte de l'alvéole de la 34. Notons les remaniements osseux sous-muqueux du côté droit. Clinical mandibulary view (2007, january), mandibulary osteonecrosis extension which affect the first premolar alveol. See the bone modification under the mucosa on the right side.

lite. Compte tenu du contexte médical général, de l'âge du patient et de l'absence de gêne fonctionnelle importante, il est décidé de ne pas intervenir chirurgicalement et de mettre en place un suivi clinique régulier.
Le contrôle de l'évolution clinique sur une année met en évidence la rapidité d'extension des lésions maxillaire et mandibulaire, la perte dentaire spontanée de 16 et 15 ainsi qu'un remodelage osseux sous-muqueux susceptible d'aboutir à de nouvelles ulcérations (Fig. 4 et Fig. 5). Dans un contexte d'hygiène bucco-dentaire très difficile et face à la suppuration persistante du foyer d'ostéonécrose maxillaire, un traitement par fluoroquinolones (Oflocet ${ }^{\circledR}$ ) est instauré 5 mois après la première consultation. Le traitement a dû être interrompu en raison de la survenue d'une tendinite, effet indésirable fréquent des quinolones. L'état physique du patient se dégradant rapidement, aucun traitement complémentaire n'est envisagé.

\section{Myélome multiple des os}

Le myélome multiple des os ou maladie de Kahler se caractérise par une prolifération maligne de plasmocytes médullaires aboutissant à la synthèse d'une immunoglobuline monoclonale, souvent de type $\lg G$ ou $\lg A$. II existe 3 stades évolutifs et l'évolution est toujours mortelle à plus ou moins long terme : la survie est supérieure à 5 ans au stade I, inférieure à 20 mois au stade III [7]. La prolifération maligne de plasmocytes s'accompagne d'une inhibition des autres cellules myéloïdes, d'où l'apparition d'une neutropénie et d'une thrombopénie. Les signes d'appel clinique sont le plus souvent osseux avec l'existence de douleurs ou de fractures spontanées. En effet, la synthèse d'OAF (osteoclast activating factor) par I'IL-1, I'IL-6, le TNFB, le TGFB et par les plasmocytes tumoraux aboutit à une stimulation ostéoclastique.

Elle se traduit radiologiquement par des plages d'ostéolyses formant des lacunes à l'emportepièce sans condensation périphérique, touchant surtout les os plats (crâne, côtes, bassin...), et biologiquement par une hypercalcémie. L'altération de l'état général est fonction du stade d'évolution du myélome. L'atteinte rénale est fréquente, favorisée par la précipitation des protéines de Bence-Jones. Les autres complications sont neurologiques (syndrome de compression médullaire, neuropathies périphériques), infectieuses (neutropénie) et hémorragiques (thrombopénie). L'amylose complique la maladie dans 5 à $10 \%$ 
des cas. Elle peut se traduire cliniquement par des manifestations cardiaques, pulmonaires, ainsi que par une xérostomie et une macroglossie.

Le traitement de la maladie de Kahler comporte une chimiothérapie associant un alkylant $\left(\right.$ Alkéran ${ }^{\circledR}$ ) et un corticoïde $\left(\right.$ Cortancyl $\left.{ }^{\circledR}\right)$. Une radiothérapie peut quelquefois s'avérer efficace pour traiter les douleurs liées à certains foyers myélomateux. Des allogreffes de moelle osseuse peuvent être envisagées ; cette indication est en général limitée aux patients âgés de moins de 45 ans ${ }^{[7,8]}$. Le traitement symptomatique des complications rénales nécessite une hydratation alcaline et la prise en charge de l'hypercalcémie. Dans certains cas d'insuffisance rénale chronique, une dialyse, voire une transplantation rénale peut être nécessaire.

La prise en charge de la douleur nécessite l'administration d'antalgique de niveau 1, 2 ou 3 . Les algies osseuses peuvent conduire à l'immobilisation du patient. Les BP sont généralement prescrits dès que le diagnostic de myélome multiple est posé, en association avec la chimiothérapie. Le plus souvent administrés par voie intraveineuse, ils ont une bonne efficacité dans le traitement des lésions osseuses qui entraînent normalement une ostéolyse et une hypercalcémie.

\section{Bisphosphonates}

Les BP, initialement appelés diphosphonates, agissent en bloquant l'activité ostéoclastique par un mécanisme encore imparfaitement connu. En diminuant les phénomènes de résorption osseuse, ils induisent une minéralisation excessive de la trame osseuse [9]. Les BP sont prescrits par voie orale ou par voie intraveineuse [10].

Dans l'ostéoporose et en prévention après pose d'une prothèse de hanche, ils sont prescrits per os. Ils sont utilisés par voie intraveineuse dans le traitement de l'hypercalcémie maligne, du myélome multiple, des métastases osseuses des cancers (seins, prostate, poumons...) et la maladie de Paget ${ }^{[11,12]}$. Les BP présentent donc de nombreuses indications et tiennent une place importante dans l'arsenal médical thérapeutique. Ils semblent responsables d'effets indésirables dans la sphère bucco-faciale [13]. II s'agit essentiellement d'ulcérations muqueuses induites par contact lors de l'administration per os et d'ostéochimionécroses des maxillaires ${ }^{[14]}$. Ces effets secondaires maxillaires sont induits principalement par les BP utilisés par voie intraveineuse, ce qui pourrait s'expliquer par le fait que leur demivie est très longue (plusieurs années). Quelques rares cas ont également été décrits avec des BP per os ${ }^{[4,15]}$. L'ostéonécrose est une complication invasive, de prise en charge délicate. La prévalence actuelle est probablement sous-estimée car la survenue est souvent tardive (supérieure à 1 an). Cliniquement l'ostéonécrose est souvent associée à des douleurs. La lésion muqueuse s'accompagne d'une exposition de l'os sous-jacent qui présente l'aspect caractéristique d'un os nécrosé, avascularisé, de consistance «sucre mouillé ». II peut exister un œdème en regard de la lésion. L'infection sous-jacente est presque systématique et s'accompagne alors d'une suppuration. L'ostéonécrose des maxillaires survient fréquemment après une intervention de chirurgie buccale. Les traumatismes muqueux d'origine prothétique sont également susceptibles d'induire une ostéonécrose des maxillaires. Dans certains cas, ces ulcérations sont apparues spontanément, sans geste iatrogène préalable.

La connaissance des mécanismes biologiques conduisant à une ostéochimionécrose pourrait peut-être permettre d'améliorer leur prévention, voire de faciliter leur traitement mais les phénomènes conduisant à ces complications sont encore méconnus. L'hyperminéralisation induite par le blocage ostéoclastique s'accompagne très probablement d'un déficit de la vascularisation favorisant aussi le risque de nécrose osseuse. Le milieu buccal constitue l'unique région corporelle où l'os peut être directement en contact avec un milieu septique en raison des traumatismes fréquents et répétés ou d'interventions chirurgicales invasives. Le contexte médical du patient, ses antécédents et ses autres traitements médicamenteux sont des facteurs supplémentaires qui favorisent le risque de survenue d'une ostéonécrose.

II n'existe pas de consensus quant à la prise en charge de patients traités par BP. Cependant, sur les bases de la prévention de l'ostéite postradique, certains auteurs ont suggéré des médecine

buccale

chirurgie

buccale

VOL. $13, \mathrm{~N}^{\circ} 3$ 2007

page 157 
médecine

buccale

chirurgie

buccale

VOL. $13, \mathrm{~N}^{\circ} 3$ 2007

page 158 démarches à suivre pour la prise en charge thérapeutique $[16,17,18]$. En prévention, avant la mise en route d'un traitement par BP, un bilan buccodentaire complet doit être réalisé par le chirurgien dentiste. La recherche de foyers infectieux y est systématique et doit se faire à l'aide d'un bilan radiographique rétro-alvéolaire long cône et d'un cliché panoramique. Ces foyers infectieux doivent être éliminés, les dents ayant un mauvais pronostic extraites, le parodonte assaini. Les prothèses amovibles doivent être ajustées et rendues atraumatiques. Les instructions d'hygiène buccodentaire sont données au patient et ce dernier doit être bien informé des risques de développement d'une éventuelle ostéonécrose. Lorsque le traitement par BP est commencé, le suivi buccodentaire doit être régulier allant d'un contrôle tous les 6 mois pour un traitement par BP per os ou tous les 4 mois pour un traitement par BP intraveineux [17]. Les foyers infectieux doivent être traités si possible de manière non invasive. Les extractions sont à éviter sauf en cas d'infection ne pouvant être traitée autrement et en cas d'atteinte parodontale avec mobilité de stade 3 . Si une extraction est nécessaire, il faut qu'elle soit le moins traumatisante possible, réalisée sans lambeau avec une régularisation des contours osseux. La colle fibrine mise en place dans l'alvéole semble être un élément favorable à la cicatrisation [9]. Une couverture antibiotique est indispensable (amoxicilline + acide clavulanique ou quinolone + métronidazole ou érythromycine + métronidazole) [17]. Elle doit commencer avant l'intervention et être prolongée au moins 10 jours afin de couvrir le premier temps de la cicatrisation. II est préférable de limiter l'utilisation des anesthésiques avec vasoconstricteurs. La prise en charge parodontale est indispensable mais doit se faire si possible sans intervention chirurgicale. La pose d'implants est contre-indiquée [19].
Si l'ostéonécrose est déclarée, la prise en charge est beaucoup plus délicate. En cas de surinfection, une antibiothérapie prolongée est indiquée. Les bains de bouche avec un produit antiseptique sont recommandés. L'indication d'une éventuelle prise en charge chirurgicale est très controversée. Le contexte général et médical du patient oriente la prise de décision. Certains auteurs préconisent une exérèse large jusqu'à la limite de l'os sain vascularisé, associée à une fermeture hermétique du site opératoire [13]. D'autres sont plus réservés et conseillent de se limiter à l'ablation des irrégularités et des séquestres osseux sans réaliser un lambeau de recouvrement $\left[{ }^{[16,20]}\right.$. Le potentiel de cicatrisation demeure toujours incertain. L'arrêt du traitement par BP ne semble pas justifié compte tenu de la demi-vie très longue de

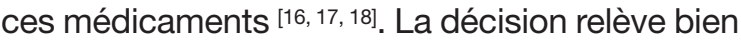
entendu du médecin prescripteur.

\section{CONCLUSION}

A partir d'une observation clinique, nous avons tenté de démontrer à quel point la prise en charge d'une ostéochimionécrose est problématique. La décision d'intervention ou d'abstention est souvent délicate à prendre et l'extension des lésions peut se faire rapidement.

Si tous les patients traités par BP ne développent pas une ostéonécrose, ils sont tous susceptibles de le faire [21]. La durée de vie des BP étant très longue, il existe potentiellement de nombreux cas à venir.

Tous les praticiens doivent être sensibilisés face à ce risque. Les conduites à tenir vis à vis de ces patients doivent donc être rigoureuses quel que soit le BP prescrit. La meilleure attitude reste la prise en charge préventive de ces patients avant l'instauration d'un traitement par BP. 


\section{RÉFÉRENCES}

1 - Lettre aux prescripteurs, Information de pharmacovigilance. Acide zolédronique / pamidronate de sodium et ostéonécrose de la mâchoire. Site internet de I'AFSSAPS ; http://agmed.sante.gouv.fr/htm/10/filltrpsc/lp050701.htm

2 - Marx RE. Pamidronate and zoledronate induced avascular necrosis of the jaws: a growing epidemic. J Oral Maxillofac Surg 2003 ; 61: 1115-7.

3 - Wang J, Goodger NM, Pogrel MA. Osteonecrosis of the jaw associated with cancer chemotherapy. J Oral Maxillofac Surg $2003 ; 61$ : 1104-7.

4 - Naveau A, Naveau B. Otseonecrosis of the jaw in patients taking bisphosphonates. Joint Bone Spine $2006 ; 73: 7-9$

5 - Magremanne M, Vervaet C, Dufrasne L, Declercq I, Legrand W, Daelemans Ph. Bisphosphonates et ostéo(chimio)nécrose maxillo-mandibulaire. Rev Stomatol Chir Maxillofac 2006 ; 107 : 423-8.

6 - Alexanian R, Dreiger R. Chemotherapy of multiple myeloma. Cancer 1984 ; 53 : 583-8.

7 - Baur Chaubert A, Delacrétaz F, Schmidt PM. Myélome multiple. Schweiz Med Forum 2005 ; 5 : 309-6.

8 - Facon T, Gastinne T, Leleu X. Myélome multiple : espoirs thérapeutiques. Rev Prat Med Gen 2003 ; 17 : 515-7.

9 - Abi Najm S, Lysitsa S, Carrel JP, Lesclous P, Lombardi T, Samson J. Ostéonécroses des maxillaires chez des patients traités par bisphosphonates. Presse Med 2005 ; 34 : 1073-7.

10 - Carrel JP, Najm SA, Lysitsa S, Lesclous P, Lombardi T, Samson J. Phosphore et bisphophonates : ou quand on oublie les leçons du passé. Med Buccale Chir Buccale $2006 ; 12: 7-14$.

11 - Soltau J, Drevs J. The role of bisphophonates in oncology. Drugs of the future $2004 ; 29: 911-21$.

12 - Body JJ, Diel IJ, Lichinitser MR, Kreuser ED, Donoff W, Gorbunova VA, Budde M, Bergstrom B. Intravenous ibandronate reduced the incidence of skeletal complications in patients with breast cancer and bone metastases. Ann Oncol 2003 ; 14 : 1399-405.
13 - Ruhin B, Agbo-Godeau S, Ben Slama L, Bertrand JC. Attention aux effets indésirables des bisphophonates : ulcération muqueuse, retard cicatriciel et ostéonécrose. Mise au point et conduite à tenir. Actual Odonto Stomatol 2006 ; $233: 7-16$.

14 - Cousty S, Duran D. Ulcération chronique dans le cadre d'une atteinte myélomateuse : à propos d'un cas. Med Buccale Chir Buccale 2006 ; 12 : 15-9.

15 - Ruggiero SL, Mehrotra B, Rosenberg TJ, Engroff SL. Osteonecrosis of the jaws associated with the use of bisphosphonates : a review of 63 cases. J Oral Maxillofac Surg 2004 ; 62 : 527-34.

16 - Hugentobler M, Richter M. Proposition d'un algorithme de prise en charge médico-dentaire pour les patients traités par bisphophonates. Rev Stomatol Chir Maxillofac 2006 ; 107 : 441-4.

17 - Marx RE, Sawatari Y, Fortin M, Broumand V. Bisphophonate-induced exposed bone (osteonecrosis/osteopetrosis) of the jaws: risk factors, recognition, prevention and treatment. J Oral Maxillofac Surg 2005 ; 63 : 1567-75.

18 - Ruggiero S, Gralow J, Marx RE, Hoff AO, Schubert MM, Huryn JM. Pratical guidelines for the prevention, diagnosis and treatment of osteonecrosis of the jaw in patients with cancer. J Oncology Pract 2006 ; 2 : 7-14

19 - Stark WJ, Epker BN. Failure of osseointegrated dental implants after diphosphonate therapy of osteoporosis: a case report. J Oral Maxillofac Implants 1995 ; 10 : 74-8

20 - Badros A, Weikel D, Salam A, Goloubeva O, Schneider A, Rapoport A, Fenton R, Gahres N, Sausville E, Ord $\mathrm{R}$, Meiller T. Osteonecrosis of the jaw in multiple myeloma patients : clinical features and risk factors. J Clin Oncol 2006 ; 24 : 945-52.

21 - Durie BG, Katz M, Crowley J. Osteonecrosis of the jaw and bisphosphonates. N Engl J Med 2005 ; 353 : 99102. \begin{tabular}{l}
$\begin{array}{l}\text { médecine } \\
\text { buccale } \\
\text { chirurgie } \\
\text { buccale }\end{array}$ \\
\hline VOL. $13, N^{\circ} 3$ \\
2007
\end{tabular}

page 159 